\title{
Guidelines for Authors
}

\section{These guidelines are available on-line at http://www.socgenmicrobiol.org.uk}

\section{SUBMISSION OF PAPERS FOR PUBLICATION}

Send:

1 Three copies of the manuscript, doublespaced throughout.

2 One printers' set of any line drawings, plus three reviewers' sets.

3 One printers' set of any halftone photographs, plus three reviewers' sets (not photocopies).

4 Two copies of any papers cited as 'accepted for publication' or 'in press'.

5 Written permission for any personal communications.

\section{To:}

Microbiology Editorial Office, Marlborough House, Basingstoke Road, Spencers Wood, Reading RG7 1AE, UK.

\section{General}

1 Microbiology publishes original work on microorganisms in the laboratory and in their natural environments, and is particularly concerned with fundamental studies. Papers are published in the following subject areas: Development and Structure, Physiology and Growth, Biochemistry, Bioenergetics and Transport, Genetics and Molecular Biology, Genome Analysis, Biotechnology, Environmental Microbiology, Plant-Microbe Interactions, Pathogenicity and Medical Microbiology, Antigens and Immunity, and Systematics. Guidelines on the presentation of Genome Analysis and Systematics papers may be obtained from the Editorial Office.

Invited review articles are also published. Authors interested in publishing such papers should write to the Editor-in-Chief at the Editorial Office.

Short Communications as such are not published, but there is in principle no lower limit on the length of papers, provided that they are of an appropriate scientific standard.

Microbiology Comment is a correspondence section where readers can briefly communicate personal observations and opinions more informally than in a conventional paper. See any recent Comment section for more detailed guidelines. Contributions for this section should be sent to the Editor-in-Chief at the Editorial Office.

2 Papers are considered for publication on the understanding that (a) they report unpublished work that is not under consideration for publication elsewhere; (b) all named authors have agreed to the submission; and (c) if the paper is accepted for publication in Microbiology, the authors (or other copyright holder) will transfer to the Society for General Microbiology the copyright of the paper, which will then not be published elsewhere in the same form, in any language, without the consent of the Society. If a paper is rejected, only one copy and the original figures will normally be returned to the author; the other copies will be destroyed unless their return was requested at the time of submission.

3 Authors should wait until a piece of work is rounded off and a comprehensive paper can be written, rather than write a series of papers on the same subject as the results come to hand.

4 There are no page charges. Authors receive 50 offprints free; further copies may be purchased in multiples of 50 .

\section{Editorial handling of papers}

Submitted papers are sent by the Editorial Office to an Editor or Member of the Editorial Board (MEB) with appropriate expertise, who is responsible for making the decision on acceptability. The Editor or MEB may act as a referee him/herself, but will normally send the paper to at least one independent referee. MEBs may additionally obtain an Editor's opinion before reaching a decision. If the paper requires relatively minor revision, the Editor or MEB will normally write to the author directly. In the case of major revision, rejection or acceptance, the letter and any reports will usually come from the Editorial Office.

Papers returned for revision that have not been resubmitted within 28 days of the author receiving the request for revision are treated as new submissions and given a new date of receipt.

\section{Cover illustrations}

The Editors welcome the submission of pictures for possible use on the front cover, and will pay $£ 50$ towards expenses for each one used. Pictures need not be linked with a paper in the journal. A picture that is linked with a paper must not be the same as a figure in the paper. 


\section{Form of papers submitted for publication}

What to send. Manuscripts should be submitted in triplicate, and be double-spaced throughout. Three sets of each figure must be included for use by the reviewers (for halftone figures the copies should be photographic prints, not photocopies), and an additional set of figures should be provided for use by the printers.

The paper must be written in clear and concise English, normally in the past tense, and should normally comprise: (a) Title page, including keywords;

(b) Summary; (c) Introduction; (d) Methods; (e) Results;

(f) Discussion, with Conclusions if appropriate;

(g) Acknowledgements, if any; (h) References. A Theory section and Appendices may be included. Where appropriate, the Results and Discussion may be combined. Figures and tables should be selected to illustrate points that cannot easily be described in the text.

Submission of papers on disk. Accepted papers are copyedited as word-processor files wherever possible, so authors are asked to provide their paper in this form on a disk when they submit the revised version. Hard copies must also be provided (see above). The version of the paper on the disk must exactly match the final, accepted version of the paper. If any differences are noticed, the hard copy will be taken as correct.

General style and layout. Authors should consult a recent issue of the journal for guidance on the layout of headings, tables, etc. More detailed guidance on the presentation of individual sections is given below. This includes some hints on how to improve the impact of a paper by appropriate presentation.

Title page. This should carry the following information.

- The title of the paper. The title should aim to attract readers by including topical keywords and by alluding to the interesting conclusions of the paper. A title that emphasizes the main conclusions, or poses a question, has more impact than one that just describes the nature of the study.

- A short 'running title', of not more than 50 characters (including spaces), for use as a headline.

- Keywords: a maximum of five words or short phrases.

- The subject category for the contents list - the categories are listed above under General, point 1.

- The names of the authors. Each author may use one given name in full. The author for correspondence must be clearly indicated.

- The name and address of the laboratory or laboratories where the work was done, and present addresses of authors who have since moved.

- Telephone and fax numbers and, if available, an e-mail address for the corresponding author.
- If appropriate, a footnote defining any non-standard abbreviations. A list of abbreviations not requiring definition is given on $\mathbf{p}$. $\mathbf{x}$.

Summary. This is likely to be read by more people than the full paper, and many abstracting services use authors' summaries without modification. It is therefore important that this section is clear and comprehensible in its own right. References should not be cited, and any abbreviations used must be defined. The summary should if possible introduce the subject in the first sentence and present the main conclusion in the last sentence: when someone is skimming a block of text, the first and last sentences receive the most attention.

Introduction. This should state the objectives of the work when it was undertaken, but should not contain a detailed summary of the results. Authors should not assume that all readers will know why an area is worth studying. They should briefly make this clear at the beginning of the introduction.

Methods. Sufficient detail should be provided to allow the work to be repeated. The suppliers of chemicals and equipment should be indicated if this may affect the results. Suppliers' addresses should not be given unless this is considered essential for a particular reason.

Results. There should be sufficient subheadings to make clear how the work was organized, what the key questions being addressed were, how one experiment led to another, and perhaps what conclusions were reached. A reader should gain a clear picture of the work from the subheadings.

Reproducibility of results. This should be indicated. It should be stated how many times an experiment was repeated and whether means or representative results are shown. Variability should be indicated statistically wherever possible; when error terms are given, the measure of dispersion and the number of observations should be stated. Statistical techniques used must be specified, and where necessary they should be described fully or a reference given. If results are expressed as percentages, the absolute value corresponding to $100 \%$ should be stated.

Discussion. This should not recapitulate the results, and should not be too long. Excessive discussion of few facts often conveys an impression of poor science.

Subheadings should be used where appropriate, to highlight the points under discussion. It may be helpful to list the main conclusions at the end. A combined Results and Discussion section is encouraged where appropriate.

References. References in the text should be cited as follows: two authors, Smith \& Jones (1996) or (Smith \& Jones, 1996); three or more authors, Smith et al. (1996) or (Smith et al., 1996). References to papers by the same author(s) in the same year should be distinguished in the 
text and the reference list by the letters a, b, etc. (e.g. 1996 a or $1996 \mathrm{a}, \mathrm{b})$.

References at the end of the paper must be given in alphabetical order, except for papers with three or more authors, which should be listed in chronological order after any other papers by the first author. References must include the title of the paper as well as both initial and final page numbers. Titles of journals should be abbreviated according to the system followed by Index Medicus and Biological Abstracts. No stops should be used after abbreviated words. References to books should include year of publication, title (in full), edition, editor(s) (if any), town of publication and publisher, in that order. When the reference is to a particular part of a book, the inclusive page numbers and, if appropriate, chapter title must be given. Examples of journal and book references are given on $\mathrm{p} . \mathrm{x}$.

Only papers accepted for publication but not yet published may be cited as 'in press' in the reference list and must be accompanied by the name of the journal. Two copies of relevant papers cited as 'in press' should be enclosed with the submitted manuscript. References to papers not yet accepted should be cited in the text as unpublished results, giving the surname(s) and initials of the author(s). Such papers should not appear in the list of references.

Written permission for any personal communications or citations of other workers' unpublished results must be provided at the time of submission.

Tables. These should be broadly comprehensible without reference to the text, but it is not necessary to repeat detailed descriptions of methods, etc. The symbols * $† \ddagger$ $\mathbb{S} \| \boldsymbol{I}$ should be used for footnotes, rather than superscript letters or numbers. When results are expressed as percentages, the absolute value(s) corresponding to $100 \%$ must be stated. Statements of reproducibility should be included (see Reproducibility of results, p. vi).

Figures. These must be selected to illustrate specific points. They should not be used to present results that can be described by a brief statement in the text. Legends should be typed double-spaced on a separate sheet. The points outlined above for tables regarding comprehensibility, relative values and reproducibility also apply to figures and their legends.

Line drawings. These should be of a quality suitable for direct reproduction and approximately twice the size that they will appear. The maximum printed size, including lettering and legends, is $176 \times 235 \mathrm{~mm}$. Line thicknesses and symbol sizes should be sufficient to allow for reduction (normally about $0.4 \mathrm{~mm}$ and $2-3 \mathrm{~mm}$, respectively). The preferred symbols for graphs are $\bigcirc$, $\boldsymbol{\bullet}, \square, \boldsymbol{\square}, \triangle, \boldsymbol{\Delta}, \nabla, \boldsymbol{\nabla}$. Graph lines should not appear inside open symbols. Where possible, the same symbol should be used for the same quantity in different figures. The scale-marks on graphs should be inside the axes.
Tints (i.e. shading made up of fine dots) should not be used in line drawings, because they do not reproduce clearly when printed in the journal.

Three reviewers' copies of each figure must be supplied. A top set of figures must also be provided for use by the printers.

Bar diagrams. Simple bar diagrams reporting only a few values are normally unnecessary; the data should instead be given in a table or in the text. It is editorial policy not to publish bar diagrams with 'three-dimensional' bars unless there is a specific justification for their use. Tints should not be used as shading for bars (see above).

Sequence data. Figures showing full gene sequences are not normally published (see p. ix). If included, figures representing nucleotide or amino acid sequences should be in high-quality camera-ready form with numbering of nucleotides or amino acid residues at appropriate intervals. Tints should not be used to highlight parts of sequences. The layout should be designed to fit either the full width of the page $(176 \mathrm{~mm})$ or a single column $(84 \mathrm{~mm})$. For adequate legibility, the height of the characters should be not less than 1.5-2 mm (or 6-8 point). For printing at full page width with this size of type, a layout with $80-100$ nucleotides per line is appropriate (or 60-70 if there are spaces between the codons). For a single-column layout, 50-60 nucleotides per line is about right. The spacing between the lines of sequence should be as close as is consistent with clarity. Note that sequence data must be submitted to GenBank, EMBL or DDBJ (see p. ix).

Photographs. These should be well-contrasted prints and approximately final size (maximum width $176 \mathrm{~mm}$ ). For photomicrographs, magnification should be shown by a bar marker. Photographs may be grouped to form a composite picture (maximum final size $176 \times 235 \mathrm{~mm}$, including space for legend); to avoid loss of definition the component parts should be submitted as separate prints, rather than being rephotographed in the composite arrangement. If images have been digitally generated, the hardware and software used must be stated.

Three reviewers' copies of each photograph must be supplied; these should be photographic prints, not photocopies. A top set of prints must also be provided for use by the printers.

Scanned 'photographs'. Authors' printed output from digitally generated scans does not reproduce well in the journal. The 'screening' process used by the printers to produce halftone illustrations converts the image into a pattern of dots. When this process is applied to an image that already consists of dots, as for scans, the two dot patterns often interfere and produce unacceptable results.

To avoid these problems, authors may submit their scans in electronic form. For this purpose, scans must be saved as 'raw' TIFF files of the images as output by the scanner. Labels, pointers, etc., must not be added to 
these files: they should instead be indicated on a printout (this is to avoid problems of incompatibility between authors' and printers' software). Graphics files should be supplied on a separate disk(s) from the text of the paper, with the filename(s) clearly indicated. Four hard-copy printouts of each figure must be supplied, one unlabelled and the others with the positions of any labels clearly marked. The printers will attempt to use all graphics sent to them on disk, but they cannot guarantee to do this. If the graphics files cannot be used, the printers will use the unlabelled printout as artwork and the labelled printout as a guide; the printouts should therefore be of the highest possible resolution.

Colour photographs. These are accepted, at the discretion of the Editors, if they are essential for an understanding of the work described. The printers can produce blackand-white photographs from colour prints if necessary, but this is not recommended.

\section{Quantities, units and symbols}

The recommended SI units should be used. For guidance, see Quantities, Units and Symbols, published by the Royal Society, 6 Carlton House Terrace, London SW1Y 5AG, UK, and Units, Symbols and Abbreviations, published by the Royal Society of Medicine, 1 Wimpole Street, London W1M 8AE, UK.

\section{Chemical and biochemical nomenclature}

Authors should follow the recommendations of IUPAC for chemical nomenclature, and those of the Nomenclature Committee of IUBMB and the IUPAC-IUBMB Joint Commission on Biochemical Nomenclature for biochemical nomenclature. A summary of nomenclatural recommendations, with references, is given in the Biochem J Instructions to Authors (printed in the first issue of the Biochem J each year and also available from Portland Press, 59 Portland Place, London W1N 3AJ, UK). The recommendations are given in full in Compendium of Biochemical Nomenclature and Related Documents, 2nd edn (1992), London: Portland Press.

\section{Absorbance, optical density, and attenuance}

The term absorbance, $A$, should be used for the quantity $\log \left(I_{0} / I\right)$ in UV and visible absorption spectrophotometry of samples in which there is negligible scattering or reflection of light. If scattering is considerable, as in spectrophotometric measurements of microbial biomass, the term optical density, OD (or attenuance, $D$ ), should be used; the path length of the cell or cuvette, and the make and model of the spectrophotometer must be specified, because optical design dramatically influences such measurements. If a sample is diluted prior to measuring optical density, the dilution and the diluent should be stated. Readings obtained with instruments designed for turbid samples, such as nephelometers or Klett meters, should be reported in appropriate units. Whenever $A, \mathrm{OD}$ or $D$ is used, the wavelength (in $\mathrm{nm}$ ) of the incident light must be specified (e.g. $A_{280}, \mathrm{OD}_{600}$ ).

\section{Enzyme nomenclature}

The system published in Enzyme Nomenclature (1992), London \& New York: Academic Press, is used. Enzyme Commission numbers should be given where appropriate.

\section{Description of strains}

A source (name and brief address) or reference should be given for each strain used. Authors are encouraged to deposit important strains in a recognized culture collection and to refer to the collection and strain number in the paper.

\section{Nomenclature of micro-organisms}

The correct name of the organism, conforming with international rules of nomenclature, must be used; if desired, synonyms may be added in parentheses when the name is first mentioned. Names of bacteria must conform with the current Bacteriological Code and the opinions issued by the International Committee on Systematic Bacteriology. Names of algae and fungi must conform with the current International Code of Botanical Nomenclature. Names of protozoa must conform with the current International Code of Zoological Nomenclature. Descriptions of new species should not be submitted unless a specimen (normally a live culture) has been deposited in a recognized culture collection and it is designated as a type strain in the paper.

The following may be found useful:

Bergey's Manual of Systematic Bacteriology. Baltimore: Williams \& Wilkins. Vol. 1 (1984), edited by N. R. Krieg \& J. G. Holt; vol. 2 (1986), edited by P. H. A. Sneath, N. S. Mair, M. E. Sharpe \& J. G. Holt; vol. 3 (1989), edited by J. T. Staley, M. P. Bryant, N. Pfennig \& J. G. Holt; vol. 4 (1989), edited by S. T. Williams, M. E. Sharpe \& J. G. Holt.

Approved Lists of Bacterial Names (1980). Edited by V. B. D. Skerman, V. McGowan \& P. H. A. Sneath, Washington, DC: American Society for Microbiology (reprinted from Int J Syst Bacteriol 30, 225-420). An amended version, with minor corrections, was published in 1989. Supplements to the Approved Lists are published in the Int J Syst Bacteriol from time to time. A List of Bacterial Names with Standing in Nomenclature is available on the Internet (ftp://ftp.cict.fr/pub/bacterio/); see Int J Syst Bacteriol 47, 590-592.

The Yeasts, a Taxonomic Study, 3rd edn (1984). Edited by N. J. W. Kreger-van Rij. Amsterdam: Elsevier. 
Yeasts: Characteristics and Identification, 2nd edn (1990). By J. A. Barnett, R. W. Payne \& D. Yarrow. Cambridge: Cambridge University Press.

\section{Genetic nomenclature}

The following proposals should be adhered to wherever possible. Bacteria: Demerec, M. et al. (1966) Genetics 54, 61-76 [also J Gen Microbiol (1968), 50, 1-14]. Plasmids: Novick, R. P. et al. (1976) Bacteriol Rev 40, 168-189. Yeasts: Sherman, F. (1981) In The Molecular Biology of the Yeast Saccharomyces. I. Life Cycle and Inberitance, pp. 639-640 (edited by J. N. Strathern et al. New York: Cold Spring Harbor Laboratory). Aspergillus nidulans: Clutterbuck, A. J. (1973) Genet Res 21, 291-296.

Neurospora crassa: Neurospora Newsl (1978), 25, 29.

\section{Ethics of human and animal experimentation}

Papers describing any experimental work with humans should include a statement that the Ethical Committee of the institution in which the work was done has approved it, and that the subjects gave informed consent to the work.

Experiments with animals should be done in accordance with the legal requirements of the relevant local or national authority. Procedures should be such that experimental animals do not suffer unnecessarily. Papers should include details of the procedures and of anaesthetics used.

The Editors will not accept papers where the ethical aspects are, in their opinion, open to doubt.

\section{Deposition of data}

Authors are encouraged to use the Supplementary Publication Scheme of the British Library Document Supply Centre to deposit detailed information supplementing papers published in the journal (e.g. extensive tables of taxonomic data). Details are available from the Editorial Office.

\section{Papers describing solely the purification and/or characterization of enzymes}

It is editorial policy not to publish papers on the above subject(s) unless they describe some particular aspect that is of significant novelty and of clear relevance to microbiology. Examples of the types of work that would be considered appropriate for publication in Microbiology are: the purification of a previously uncharacterized enzyme; a description of unique properties of an established class of enzyme; the development of a new and broadly applicable purification technique; or a report of properties of direct relevance to the functions or application of the producing micro-organism.
The significance of the work should be indicated in the title: uninteresting titles such as 'Purification and characterization of a $\beta$-glucanase from Bacillus subtilis' should be avoided.

Descriptions of well-known enzymes that are already known to be produced by a number of micro-organisms are not appropriate for Microbiology.

\section{Papers reporting original nucleotide or amino acid sequence data}

Such papers should be accompanied by substantial additional experimentation to characterize the gene(s) and products(s) concerned, and substantial computer analysis. A sequence alone is unlikely to be acceptable. Microbiology will not normally publish DNA sequences from double-stranded genomes unless both strands have been sequenced independently.

Microbiology will no longer publish figures whose principal function is to present primary sequence data, since the data can be accessed through the databases. To merit publication, sequence figures must be justified by the additional annotation they present; they should normally be limited to regions of particular interest. Sequence alignments of nucleic acids and proteins will be acceptable provided they make a significant point. To assist the reviewers, authors must send three hard copies of the complete sequence covered by their paper at the time of submission.

Papers reporting new sequence data will not be published unless the sequence has an accession number from one of the public databases. Instructions on how to submit data to GenBank, EMBL, the DNA Database of Japan (DDBJ) or PIR can be obtained from the addresses below.

Submitted manuscripts containing sequence data should include, on the title page, the footnote "The GenBank [or EMBL/DDBJ/PIR] accession number for the sequence reported in this paper is X00000'.

GenBank: GenBank Submissions, National Center for Biotechnology Information, Bldg 38A, Rm 8N-803, 8600 Rockville Pike, Bethesda, MD 20894, USA.

e-mail:gb-sub@ncbi.nlm.nih.gov

Tel: +1 301496 2475. Fax: +1 3014809241.

EMBL: EMBL Nucleotide Sequence Database, European Bioinformatics Institute, Hinxton Hall, Hinxton, Cambridge CB10 1RQ, UK. e-mail: datasubs@EBI.AC.UK

Tel: +44 1223 494401. Fax: +441223494468.

DDBJ : DDBJ, National Institute of Genetics, Mishima, Shizuoka 411, Japan. e-mail: ddbjsub@ddbj.nig.ac.jp

PIR: PIR, NBRF, 3900 Reservoir Road NW, Washington, DC 20007-2195, USA. e-mail: PIRMAIL@NBRF.Georgetown.Edu Tel: +1 2026872121 . Fax: +12026871662. 


\section{At-a-glance style guide}

Abbreviations: to be listed on title page, and to be defined at first mention in both Summary and main text. The following need not be defined: aa ; ACES; ADA; ADP, cAMP, ATP, etc.; AIDS; BES; Bicine ; bp; BSA; CAPS ; c.f.u.; CHAPS ; CHES; CIE ; CM-cellulose; CoA; c.p.m.; Da ; DEAE-cellulose; DMSO; DNA, cDNA, CCC DNA, dsDNA, rDNA, ssDNA, DNase; DNP; d.p.m., d.p.s.; DTT; ED 50 ; EDTA, EGTA ; ELISA; EMS; e.o.p.; EPR or ESR ; FITC; FPLC; GC or GLC; GSH, GSSG ; HEPES ; HEPPS ; HPLC; i.d.; IEF ; IgG, IgM, etc. ; IPTG; IR ; kb, kbp; LD 50 ; LPS; mAb; MES; MIC; m.o.i.; MOPS ; MS ; NAD, NADP ; NMR ; nt ; NTG; ONPG; ORF; PAGE ; PBS ; PCR ; PEG; PFGE; p.f.u.; $P_{i}, P_{i}$; PIPES ; PMSF ; ppGpp, pppGpp; p.p.m.; p.s.i.; PVDF; Py-GC, Py-MS; RBS; RFLP; RNA, mRNA, rRNA, tRNA, RNase; r.p.m.; RT-PCR; SDS, SDS-PAGE; TCA; TES; TLC; Tricine, Tris; UV; X-Gal.

Compound units: $\mu \mathrm{g} \mathrm{ml}^{-1}$ not $\mu \mathrm{g} / \mathrm{ml} ; 10 \mu \mathrm{g}$ ampicillin $\mathrm{ml}^{-1}$ not $10 \mu \mathrm{g} \mathrm{ml}^{-1}$ ampicillin.

Concentration: given in $\mathrm{g} \mathrm{l}^{-1}$, etc., or molarity, $\mathrm{M}$, not normality, $N$. The term '\%' should be defined as ' $w / v$ ', ' $v / v$ ' or ' $w / w$ ' if this is necessary to avoid ambiguity.

Figures: refer to in text as Fig. 1(a) not FIG 1A, Figure $1(\mathrm{~A})$, etc.; or as (Fig. 1a) not (Figure 1 A). Multipart figures should be labelled (a), (b), etc., not A, (A) or B, (B).

Genes and genotypes: gyrA not gyrA; $\arg -1$ not $\arg 1$ or $\arg 1$, etc.

Molecular mass in $\mathrm{Da}$, e.g. $31500 \mathrm{Da}$ or $31.5 \mathrm{kDa}$; or relative molecular mass, $\boldsymbol{M}_{\mathrm{r}}$, without units, e.g. $M_{\mathbf{r}}$ 31500 , not molecular weight. In table headings and figure axes, for values $>1000$ use $\mathrm{kDa}$ or $10^{-3} \times M_{\mathrm{r}}$.

Pressure: $\mathrm{Pa}$ preferred; if given in p.s.i., equivalent in $\mathrm{Pa}$ must be given ( 1 p.s.i. $=6.9 \mathrm{kPa}$ ).

Radioactivity: $\mathrm{Bq}$ preferred; if given in $\mathrm{Ci}$, equivalent in $\mathrm{Bq}$ must be given $\left(1 \mathrm{Ci}=3.7 \times 10^{10} \mathrm{~Bq}\right)$; may also be expressed as d.p.s. ( 1 d.p.s. $=1 \mathrm{~Bq}$ ) or as c.p.m.

References: In the text: note ' $\&$ ' not 'and', e.g. Pasta \& Sicard (1996) not Pasta and Sicard (1996), or (Pasta \& Sicard, 1996) not (Pasta and Sicard, 1996); note italic 'et al.' with three or more authors, e.g. Santos et al. (1996) or (Santos et al., 1996).

In the list: note space between authors' initials; abbreviated journal titles in italics without full stops (one-word titles in full); use of bold type for authors, date and volume number; comma not colon after volume number; book publisher's city or town and state, followed by name.

Sample journal references:

Pasta, F. \& Sicard, M. A. (1996). Exclusion of long heterologous insertions and deletions from the pairing synapsis in pneumococcal transformation. Microbiology 142, 695-705.

Santos, J. A., López-Dlaz, T., Garcla-Fernández, M.-C., GarcíaLópez, M.-L. \& Otero, A. (1996). Effect of a lactic starter culture on the growth and protease activity of Aeromonas bydrophila. $J$ Appl Bacteriol 80, 13-18.

Sample reference to a whole book:

Sambrook, J., Fritsch, E. F. \& Maniatis, T. (1989). Molecular Cloning: a Laboratory Manual, 2nd edn. Cold Spring Harbor, NY: Cold Spring Harbor Laboratory.

Sample reference to a book chapter or section:

Romano, A. H. \& Saier, M. H., Jr (1992). Evolution of the bacterial phosphoenolpyruvate: sugar phosphotransferase system. I. Physiological and organismic considerations. In The Evolution of Metabolic Function, pp. 171-204. Edited by R. P. Mortlock. Boca Raton, FL: CRC Press.

Restriction enzymes: e.g. EcoRI not EcoRI, etc.; HindIII not HindIII, HindIII, Hind III, etc.

Spectrophotometry: Absorbance, $A$, absorption of monochromatic light; for clear solutions or for absorbing molecules in turbid suspensions. Optical density, OD, for non-monochromatic light or for turbid suspensions (attenuance, $D$, may also be used). Wavelength and instrument must be stated.

Suppliers: name of company without Ltd, Co., Corp., etc.; no address.

Table footnote symbols: $+\ddagger \mathbb{S} \| \mathbb{S}$ in that order from left to right and top to bottom.

Units: note space between number and unit, e.g. $5 \cdot 4 \mathrm{~kb}$ not $5 \cdot 4 \mathrm{~kb} ; 37^{\circ} \mathrm{C}$ not $37^{\circ} \mathrm{C} ; 10000 \mathrm{~g}$ not $10000 \mathrm{~g}$. 